\title{
A Precision Metrology System for the Hubble Space Telescope Wide Field Camera 3 Instrument
}

\author{
Ronald W. Toland \\ NASA/Goddard Space Flight Center \\ Code 551.1 Optical Systems Alignment and Test Group \\ Greenbelt, MD 20771
}

\begin{abstract}
The Wide Field Camera 3 (WFC3) instrument for the Hubble Space Telescope (HST) will replace the current Wide Field and Planetary Camera 2 (WFPC2). By providing higher throughput and sensitivity than WFPC2, and operating from the near-IR to the near-UV, WFC3 will once again bring the performance of HST above that from ground-based observatories. Crucial to the integration of the WFC3 optical bench is a pair of 2-axis cathetometers used to view targets which cannot be seen by other means when the bench is loaded into its enclosure. The setup and calibration of these cathetometers is described, along with results from a comparison of the cathetometer system with other metrology techniques.
\end{abstract}

Keywords: cathetometer, optical metrology, calibration

\section{INTRODUCTION}

The Wide Field Camera 3 (WFC3) instrument for the Hubble Space Telescope (HST) will replace the current Wide Field and Planetary Camera 2 (WFPC2) during HST Service Mission 4 sometime in the next year. By providing higher throughput and sensitivity than WFPC2, and operating from the near-IR to the near-UV, WFC3 will once again bring the performance of HST above that from ground-based observatories.

Integration and testing of WFC3 is currently underway at NASA/Goddard Space Flight Center (NASA/GSFC). Part of this extensive process involves positioning the optical bench inside the enclosure for the original Wide Field and

Planetary Camera (WFPC1). This enclosure protects the optical bench and serves as the interface between the bench and the latches on HST. As the enclosure dictates the position of the bench with respect to the telescope, it is crucial that the bench be properly oriented in the enclosure.

To ensure proper positioning, there are several metrology references on the optical bench. A reflective plate with reticules defining the V1 axis of the telescope, the Chief Ray Target Plate (CRTP), is attached to the front of the bench. This serves as both a Cartesian coordinate and angular reference for the optical bench. Also attached to bench are three alignment cubes with reticules on each face. These serve as additional angular and Cartesian references at strategic points along the optical bench.

Figure 1 shows the layout of the metrology references on the bench. It can be seen that the three alignment cubes lie some distance inside the bench. These positions make it possible to measure the pointing directions of the cube faces through holes cut into the bench (and the enclosure holding it), but prevent measurement of the reticule locations through normal means-theodolites or laser trackers. 


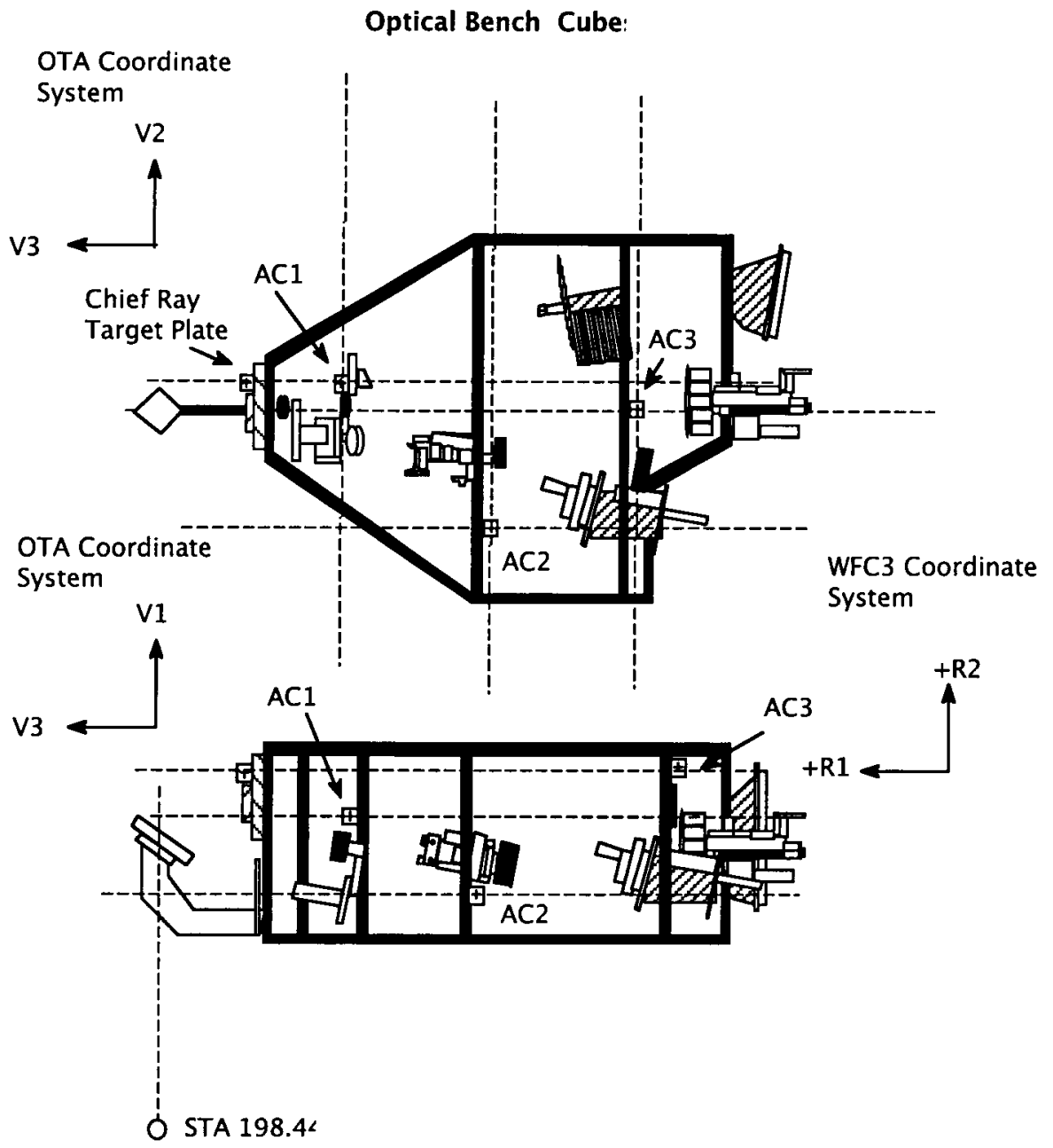

Figure 1: Position of the alignment cubes inside the WFC3 Optical Bench. 
Instead, a pair of 2 axis cathetometers must be used to ascertain the position of the cube reticules. A cathetometer-in this case-is simply an alignment telescope mounted on a 2 axis stage. An electronic encoder scale is used to read out the position of the stage along both axes. Moving the alignment telescope horizontally and vertically until the internal reticule of the telescope is aligned with the reticule on the cube face of interest and then recording the position of the telescope stage allows the reticule's position to be measured in 2 dimensions. A third dimension is added by placing a second 2-axis cathetometer at 90 degrees to the first. Figure 2 shows the layout of the cathetometers used for the integration of the WFC3 optical bench.

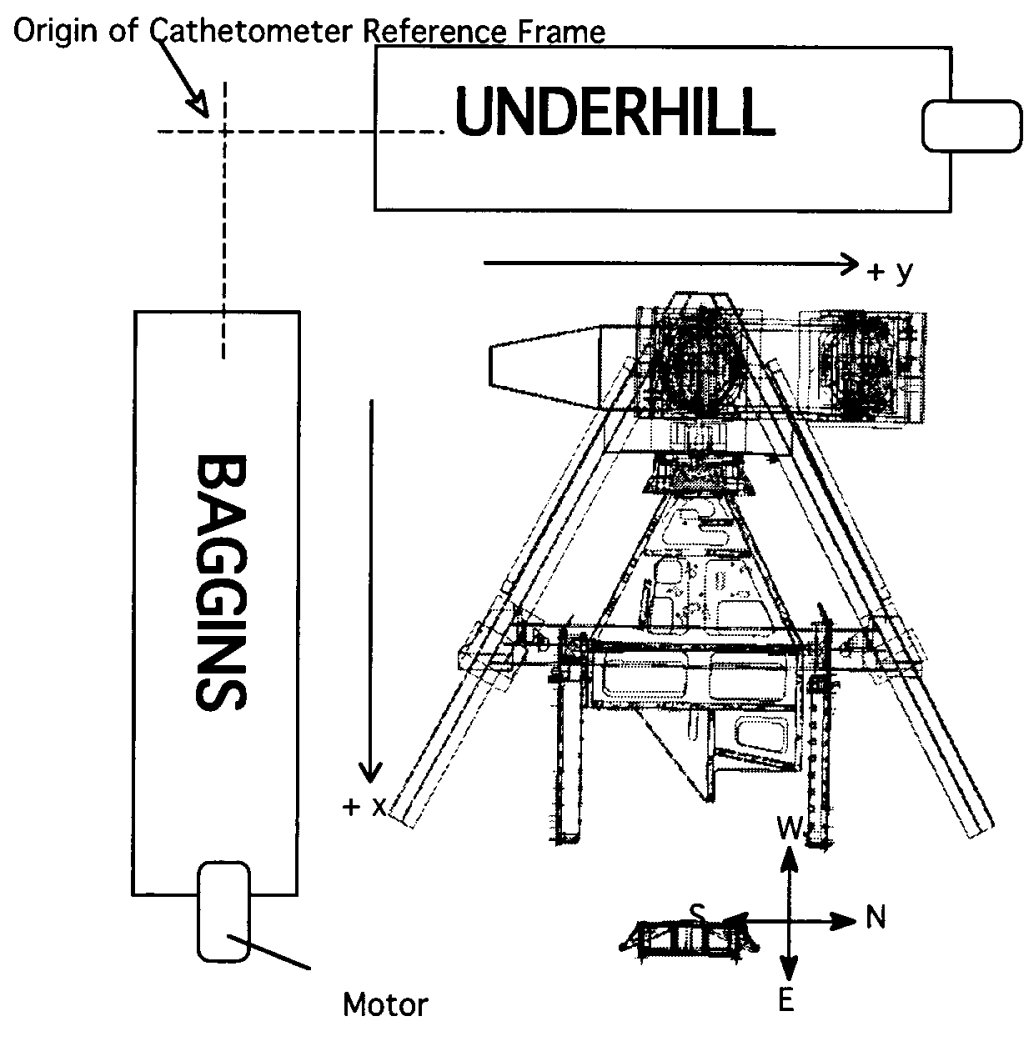

Figure 2: Orientation of the Cathetometers with respect to the WFC3 Bench.

\section{PROBLEM DEFINITION}

To be useful during integration and test, the cathetometers need to provide position measurements good to $+/-0.005$ ". Numerous errors can arise in the use of these cathetometers. If the 2 cathetometers are not placed orthogonal to each other, their measurements will not form a true coordinate system, inducing error into the measurements. If the encoders do not function properly, dropping readings occasionally, the distances between points will seem shorter than they are. There could be errors in the orthogonality of the horizontal and vertical axes of each cathetometer, errors in the pointing directions of the alignment telescopes mounted to each stage, mislevel of the cathetometer itself, or errors from motion of the stage as it moves along.

The cathetometers were oriented orthogonal to each other via theodolites and cubes placed on the side of the granite block supporting each cathetometer. Testing with a laser tracker and theodolites confirmed a general orthogonality of the horizontal and vertical axes for each cathetometer. Theodolites were again used to orient the alignment telescopes on each stage. 
However, it was found that the stage did 'wobble' significantly as it moved horizontally or vertically, inducing large errors into the measurements. This implied that each cathetometer needed to be individually calibrated, that the readouts from each encoder needed to be mapped to some accurate reference to allow use of the cathetometers within the accuracy demanded.

\section{CALIBRATION PLAN}

The problem of calibration boils down to that of knowledge. How can we know the position of the platform (and thus the alignment telescope) for all points along the axes of travel?

A set of 3 equations, described below, gives the position of the platform as a function of the electronic readout from the cathetometer itself. By knowing the angular movement of the platform as it traverses its plane of motion, the raw position given by the encoders of the cathetometer can be converted to a true position in a gravity-referenced coordinate system. The uncertainty in the calibrated position is governed by the uncertainties in the knowledge of that angular motion.

Propagating these uncertainties through the calibration equations shows the angular errors incurred by the motion of the cathetometer tower must be known to arcseconds or better for the system to achieve the required precision. In addition, these angles must repeat themselves to the same precision as they are measured. If one day measurement of the angular rotation yields one value, and another day the same measurement yields a value a full arcminute different, the angle is then only known to an arcminute, regardless of the precision of the original measurement. Care must then be taken to characterize not only the angular errors inherent in the cathetometer, but also the repeatability of those errors. As an extra precaution against failure to meet requirements, the angular errors are measured to 1 arcsecond. This gives a theoretical accuracy of less than a thousandth of an inch, and allows precise description of the repeatability of the angles measured.

Two main tools are used to take these precise measurements. A laser ranging interferometer allows measurement of the roll (pitch for the $y$ axis) and yaw angular errors for each cathetometer. It also is used to calibrate the encoders on the cathetometer, giving us a measurement of how accurately the cathetometer can report the motion of the base. As this interferometer can only measure differences between one position and another, an electronic level gives a gravity reference for the interferometer measurements and is used alone to measure the third angular axis. The interferometer has a precision of 0.01 arcseconds, and the level is precise to 0.2 arcseconds.

The question then arises of how often to make these measurements. As the angular error cannot be measured at every point along the cathetometer's length of travel, some graininess must be introduced into the measurements. At the same time, some method must be found to give the angular error at every point in the plane of the cathetometer, else the calibration is only good at the exact locations where the angles are measured.

This problem is solved with the help of an interpolation routine. By fitting a function to the data points collected and solving for the value of the angular error at the point of interest, angular data taken at a finite interval can be used to describe the entire plane. Bilinear interpolation allows a 2-D grid of angular data points with a spacing of $20 \mathrm{~mm}$ to be filled in without significant introduction of uncertainty. This interpolation scheme is both rapid and allows easy calculation of the uncertainty in the interpolated points.

Interactive Data Language (IDL) is used for both interpolation and calibration. The measured angular error data is tabulated into text files and used to interpolate the angles needed. These interpolated values are then plugged into the calibration equations to give the correct position in three dimensions for the point of interest.

\section{CALIBRATION EQUATIONS}

In describing the position of the cathetometer platform, its angular motion is broken up into three components, roll, pitch, and yaw. Roll is defined to be rotation about the $\mathrm{x}$ axis. Pitch is rotation about the $\mathrm{y}$ axis. Yaw is rotation about 
the $\mathrm{z}$ axis (vertical). Figures 2 and 3 show the orientation of the three components with respect to the axes of the cathetometer coordinate system and the origin of that coordinate system.

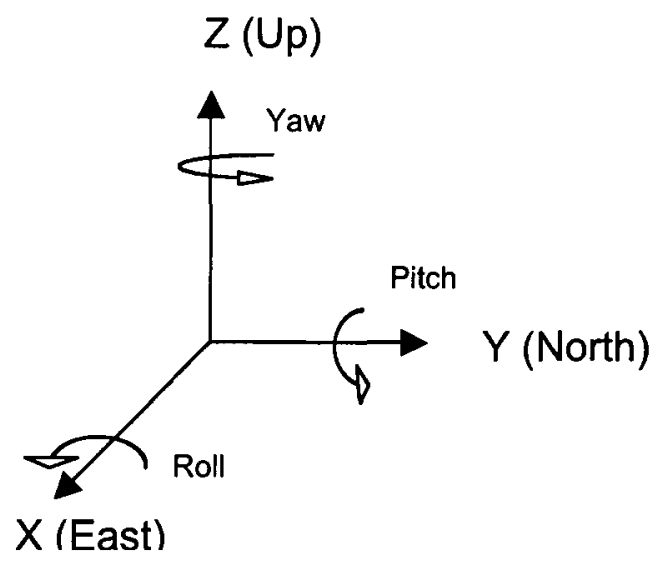

Fig. 3: Orientation of the coordinate system for the cathetometers

We seek the calibrated position along each axis in the cathetometer reference frame. Each axis has a position given by the encoders on the cathetometers, designated by the name of that axis (e.g., the variable $\mathrm{x}$ for the $\mathrm{x}$ axis). This reading is manipulated using the linear and angular calibration data to give us the calibrated position for that axis (e.g., $\mathrm{x}_{\mathrm{c}}$ ).

Linear calibration data is taken by setting up the Renishaw for linear position measurement along the axis $(x, y$, or $z)$ to be tested. The cathetometer stage is translated along the desired axis, and every $20 \mathrm{~mm}$ the position reading of the appropriate encoder and of the Renishaw is recorded. The offset between these values is then averaged and can then be used in the calibration equation as the correction factor for encoder error. In our equations it is assigned a Greek letter for each axis ( $\delta$ for the $x$ axis, $\beta$ for the $y$ axis, and $\gamma$ for the $z$ axis).

Both roll and pitch are measured with respect to the gravity vector, with yaw measured relative to the point in $(x, z)$ or $(y, z)$ that the alignment telescope for the cathetometer in question has been set to point perpendicular to the axis of travel ( $\mathrm{x}$ or $\mathrm{y}$ ). The angle for each is designated by a subscripted $\varepsilon$, with $\varepsilon_{1}$ the roll angle, $\varepsilon_{2}$ the pitch angle, and $\varepsilon_{3}$ the yaw angle.

The position along each axis is affected by two angles. For both pitch and roll, the shift along the translation axis is calculated using the corrected (via the linear calibration data) position along the z-axis as the moment arm of the motion. For yaw, the shift along the axis is calculated using the distance to the object being measured-the shift in the $x$-axis is calibrated using the $y$-axis readings, and vice-versa. The diagram below shows a sample calculation for the error in the $\mathrm{x}$-axis position caused by a shift in pitch. 


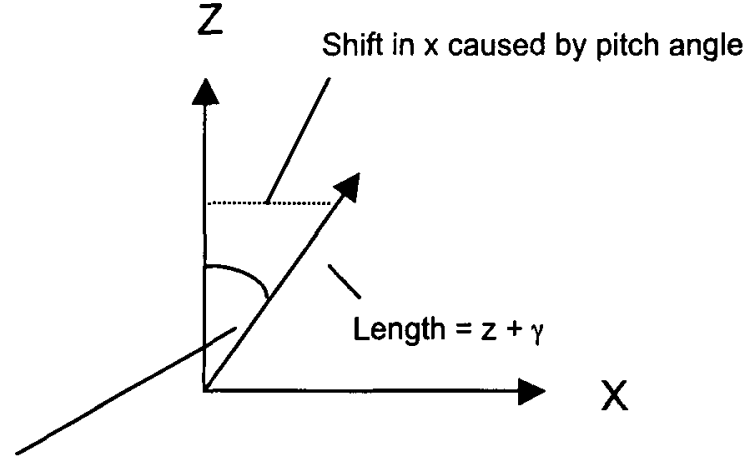

Pitch angle $\left(\varepsilon_{2}\right)$

Shift in $x=$ Length ${ }^{*} \sin \left(\varepsilon_{2}\right)=(z+\gamma)^{*} \sin \left(\varepsilon_{2}\right)$

Figure 4: Error in $\mathrm{x}$ caused by shift in pitch

Note that the intersection of the $x$ and $z$ axes in the above diagram is not necessarily the origin $(x=0)$, but represents the $x$ reading given by the encoder on the cathetometer (i.e., where the encoder thinks the cathetometer is along the $\mathrm{x}$ axis). The shift in $\mathrm{x}$ above is then the shift away from this encoder position caused by the rotation of the tower in pitch.

To correct the encoder reading for each axis, all the errors listed above must be taken into account - the offset calculated from the Renishaw linear calibration and the errors introduced by shifts in roll, pitch, and yaw. For the $\mathrm{x}$ and $\mathrm{y}$ axes, we start with the encoder position corrected with the Renishaw data then calculate the offsets generated by the appropriate angular shifts. For the $\mathrm{z}$-axis, we consider a rotation of the Renishaw-corrected $z$ distance through a pure pitch shift followed by a rotation of the resulting vector through the roll angle. The calibrated positions of each axis are given by:

$$
\begin{aligned}
& x_{c}=x+\delta+(z+\gamma)_{x} \sin \varepsilon_{2, x}+y_{c} \tan \varepsilon_{3, x} \\
& y_{c}=y+\beta+(z+\gamma)_{y} \sin \varepsilon_{1, y}+x_{c} \tan \varepsilon_{3, y} \\
& z_{c}=(z+\gamma)\left(\cos \varepsilon_{1} \cos \varepsilon_{2}\right)
\end{aligned}
$$

We see that the calibrated values for the $\mathrm{x}$ and $\mathrm{y}$ axes are coupled. To decouple them, we simply plug in the expression for $y_{c}$ into the equation for $x_{c}$ and solve for $x_{c}$. We obtain:

$$
\begin{aligned}
& x_{c}=\frac{x+\delta+(z+\gamma)_{x} \sin \varepsilon_{2, x}+\left(y+\beta+(z+\gamma)_{y} \sin \varepsilon_{1, y}\right) \tan \varepsilon_{3, x}}{1-\left(\tan \varepsilon_{3, y}\right)\left(\tan \varepsilon_{3, x}\right)} \\
& y_{c}=y+\beta+(z+\gamma)_{y} \sin \varepsilon_{2, y}+\left(\frac{x+\delta+(z+\gamma)_{x} \sin \varepsilon_{2, x}+\left(y+\beta+(z+\gamma)_{y} \sin \varepsilon_{1, y}\right) \tan \varepsilon_{3, x}}{1-\left(\tan \varepsilon_{3, y}\right)\left(\tan \varepsilon_{3, x}\right)}\right) \tan \varepsilon_{3, y}
\end{aligned}
$$

These expressions are hardly simple, but allow the $\mathrm{x}$ and $\mathrm{y}$ positions to be corrected independently-no tail-chasing here. They are also exact; the uncertainty in these calculated positions is solely from the uncertainty in the measured data.

\section{INTERPOLATION ALGORITHM}

Because of its simplicity and the ability to use propagation of error to calculate the uncertainties inherent in the method, bilinear interpolation is used as the interpolation algorithm. Angular data taken every $20 \mathrm{~mm}$ along each axis is tabulated and used as a reference in a weighted average to compute the angular error at the point of interest (the raw data point). A 
grid spacing of $20 \mathrm{~mm}$ allows the error incurred by this interpolation to be kept low and ensures the functions in-between grid points are linear and smooth.

The algorithm is summarized as follows':

Let $\mathrm{A}$ be the matrix of dimensions $\mathrm{M} \times \mathrm{N}$ holding the measured angular error values. Let $\mathrm{XI}$ be the matrix holding the positions along one axis at which $A$ was measured. Let $Y 1$ be the matrix holding the positions along the second axis at which A was measured.

We wish to find a new value $A(X, Y)$, where $X$ lies between $X 1(j)$ and $X 1(j+1)$, and $Y$ lies between $Y 1(k)$ and $Y 1(k+1)$. Letting $A 1=A(j, k), A 2=A(j+1, k), A 3=(j+1, k+1), A 4=A(j, k+1)$, we can say:

$$
\begin{aligned}
& t=\frac{(X-X 1(j))}{(X 1(j+1)-X 1(j))} \\
& u=\frac{(Y-Y 1(k))}{(Y 1(k+1)-Y 1(k))} \\
& A(X, Y)=(1-t)(1-u) A 1+t(1-u) A 2+t u A 3+(1-t) u A 4
\end{aligned}
$$

\section{CALIBRATION SETUP}

The setup for taking data with the laser-ranging interferometer is illustrated in Figure 5. The beam passes through a beamsplitter before its two components strike a pair of retroreflectors and return to be re-combined into a single beam which lands on the detector portion of the laser head. Any tilt between the two reflectors causes an optical path difference between the two beams and thus fringes at the detector. As this gives a sensitivity to shifts of less than a wavelength, the interferometer can measure angular changes of 0.01 arcsec.

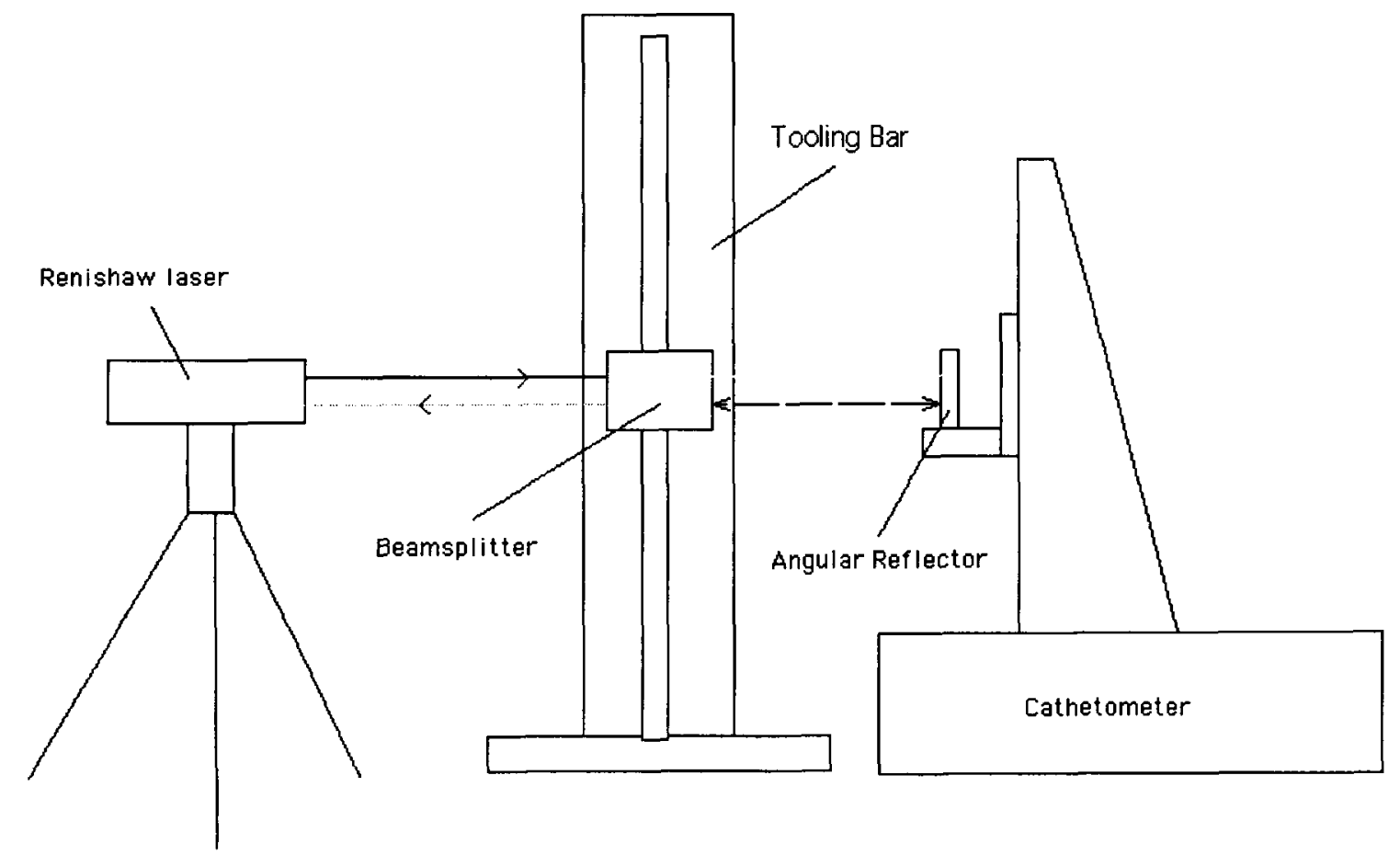

Figure 5: Laser-ranging interferometer setup to take angular data. 
In use, the interferometer is 'zeroed' at the beginning of travel for the cathetometer at a given height. It the reflector is then moved along with the tower across the length of the cathetometer's travel, with measurements of the angular shift from the start position recorded every $20 \mathrm{~mm}$. Upon reaching the end of travel, the reflector is run back to the beginning to check the repeatability of the angles measured. With this verified, the reflector is translated up $20 \mathrm{~mm}$, the interferometer is re-zeroed, and the process is repeated. Care is taken before each run to ensure the laser beam is aligned with the horizontal axis of travel of the cathetometer to prevent cosine error from affecting the measurements.

For roll (or pitch on the y axis) measurements, each horizontal line of measurements is related to those around it with the electronic level before the start of the data run. With the reflector is its initial position, the level is placed along the axis of travel and its reading recorded. This gives the angle between the platform normal at that position and the gravity vector. As the interferometer records this angle as zero, all subsequent measurements with the interferometer are shifted by the level reading to bring them into an absolute gravity reference frame. All lines measured are then related to all others, as all are measured with respect to the same reference (gravity).

As shown in Figure 6, the level is also used to measure all of the angular errors of a given axis (pitch for the $\mathrm{x}$ axis, roll for the $y$ axis). Here it is simply mounted on the platform with a C-clamp for stability. Gravity-reference readings are then taken for all points in the plane for that axis.

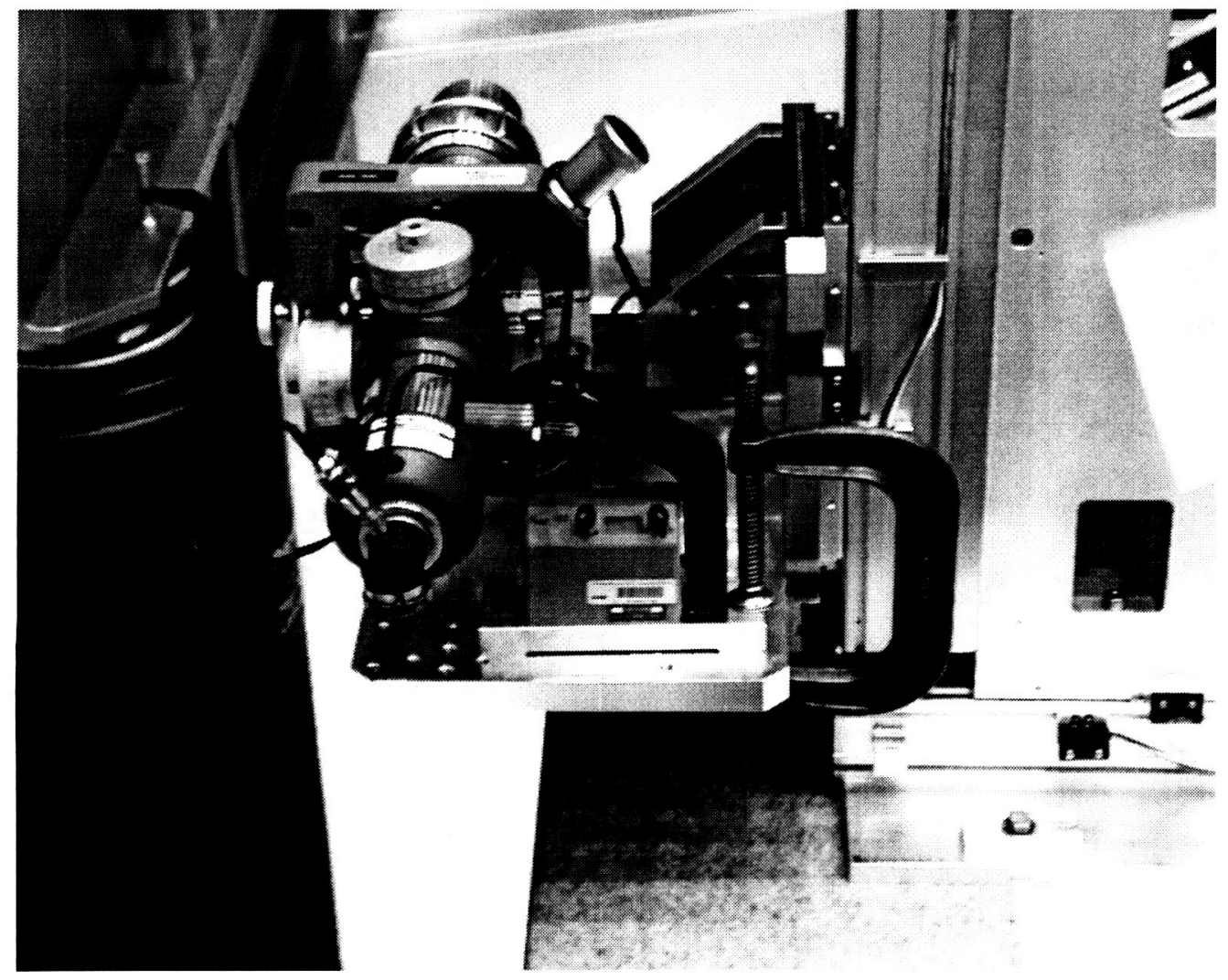

Figure 6: Electronic level setup for angular data acquisition.

After all angular errors have been recorded for all axes for both cathetometers, the data is entered into spreadsheets. There the interferometer measurements are combined with the level readings as explained above to give gravity- 
referenced angular errors at all points in the plane. The data is then converted into radians and stored in tabulated text files for later retrieval by the IDL software.

\section{INTEGRATION}

The operation of the calibrated cathetometer system can be summarized as follows. A Labview VI (developed by the author) issues commands to the cathetometer motors, moving the stage into its approximate position for target acquisition. A joystick is then used by the operator looking through the alignment telescope to fine tune the position of the telescope until it is aligned to the desired target. Retroreflectors mounted inside aluminum sphere targets are used for their increased repeatability and so the telescopes on both cathetometers, even though orthogonal to each other, can acquire the same point in space. With the telescope in place, the position of the cathetometer (given by the electronic encoders along the axes of travel) is recorded with the Labview VI. Five measurements are taken for each target. The above process is then repeated for every target of interest. The operators then switch to the second cathetometer to measure the same targets.

With the data taken and stored in text files, one for each cathetometer, the top-level IDL routine is run. The user is prompted to enter where each raw data file can be found, and the measured data is then calibrated using the extensive angular error data taken earlier. The calibrated points, along with calculated uncertainties for each, are then displayed in a table which can be saved to another text file. This text file can be imported and used by Excel or Spatial Analyzer for analysis of the gathered data.

\section{TESTING}

The system as a whole is tested by comparison to a known reference. In our case, that reference is the T-bar for the Wide Field Camera 3, the same T-bar that was used for the original Wide Field and Planetary Camera. This aluminum T-bar is the master reference gauge for the interface of the WFC3 to the Hubble Space Telescope (HST), with dimensions traceable back to the building of HST itself. A series of metrology targets, recently mechanically calibrated to 0.0002 ", is measured by the cathetometers and a laser tracker system to assess the uncertainties associated with the cathetometer measurements. Two extra targets are added to the T-bar to allow more measurement points in common between the laser tracker and the cathetometers.

The T-bar is loaded for these measurements into the Radial Instrument Alignment Fixture (RIAF), which simulates the interface to HST that WFC 3 will see on orbit. The targets are then valued by the laser tracker system, with each point measured five times for enhanced precision. The same targets are then measured by the cathetometers, again with five data points per target. To get a measure of the human error involved in the cathetometer measurements, two data sets are collected, each taken by a different operator. The data from the laser tracker and the cathetometers is then compared to the mechanical calibration data using a best-fit transformation to give a measure of the uncertainty in the cathetometer measurements.

As a further check of the uncertainty of the cathetometer measurements, targets are added to the Surrogate Wide Field Camera, a piece of tooling to simulate the size and weight of the actual optical bench. 6 targets are placed such that they can be viewed by both the cathetometers and a single laser tracker station while the surrogate camera is latched into the RIAF. These targets are then evaluated by both the laser tracker system and the cathetometers, with a third operator taking the cathetometer measurements. Again, five data points were taken for each target.

\section{RESULTS}

The data supports the conclusion that operator error plays a significant role in the cathetometer measurements. The average precision (defined as the standard deviation of the mean for the 5 data points taken per target) of the measurements taken by Operator 1 is 0.00075 ", while that for Operators 2 and 3 is twice as large. Figure 7 shows the data taken by Operators 1 and 2, as well as the average and standard deviation of the mean for each target. Because of its high precision, the data from Operator 1 is used in the analysis of the T-bar targets. 


\begin{tabular}{|c|c|c|c|c|c|c|c|}
\hline & & & Operator 1 & & & Operator 2 & \\
\hline Point & & $\mathrm{X}$ & $\mathrm{Y}$ & $\mathrm{Z}$ & $\bar{X}$ & $\mathrm{Y}$ & $\mathrm{Z}$ \\
\hline \multirow[t]{3}{*}{ RIAF C1 } & Average & 98.7694 & 67.7693 & 14.2863 & 98.7673 & 67.7971 & 14.2893 \\
\hline & Stnd Dev & 0.00022 & 0.00035 & 0.00045 & 0.00128 & 0.00129 & 0.00258 \\
\hline & $\begin{array}{l}\text { Stnd Dev } \\
\text { Mean }\end{array}$ & 0.000096 & 0.00016 & 0.00020 & 0.00057 & 0.00058 & 0.00116 \\
\hline \multirow{3}{*}{$\begin{array}{l}\text { TBAR } \\
\text { THB5 } \\
\end{array}$} & Average & 106.4856 & 80.7123 & 15.3852 & 106.4828 & 80.7117 & 15.3883 \\
\hline & Stnd Dev & 0.00051 & 0.00081 & 0.00032 & 0.00332 & 0.00098 & 0.00247 \\
\hline & $\begin{array}{l}\text { Stnd Dev } \\
\text { Mean }\end{array}$ & 0.00025 & 0.00041 & 0.00016 & 0.00166 & 0.00049 & 0.00123 \\
\hline \multirow{3}{*}{$\begin{array}{l}\text { TBAR } \\
\text { THB3 } \\
\end{array}$} & Average & 106.4990 & 118.0590 & 15.3869 & 106.4960 & 118.0551 & 15.3888 \\
\hline & Stnd Dev & 0.00023 & 0.00050 & 0.00018 & 0.00067 & 0.00275 & 0.00290 \\
\hline & $\begin{array}{l}\text { Stnd Dev } \\
\text { Mean }\end{array}$ & 0.00011 & 0.00025 & 0.000090 & 0.00034 & 0.00137 & 0.00145 \\
\hline \multirow{3}{*}{$\begin{array}{l}\text { RIAF } \\
\text { Center C }\end{array}$} & Average & 63.1383 & 93.1734 & 15.0464 & 63.1369 & 93.1737 & 15.052 \\
\hline & Stnd Dev & 0.00038 & 0.00039 & 0.00014 & 0.00106 & 0.00065 & 0.00326 \\
\hline & $\begin{array}{l}\text { Stnd Dev } \\
\text { Mean }\end{array}$ & 0.00019 & 0.00020 & 0.000070 & 0.00053 & 0.00032 & 0.00163 \\
\hline \multirow[t]{3}{*}{ RIAF C2 } & Average & 64.3678 & 90.6931 & 36.2945 & 64.3725 & 90.6922 & 36.2962 \\
\hline & Stnd Dev & 0.00113 & 0.00022 & 0.00050 & 0.00466 & 0.00152 & 0.00324 \\
\hline & $\begin{array}{l}\text { Stnd Dev } \\
\text { Mean }\end{array}$ & 0.00050 & 0.000096 & 0.00022 & 0.00209 & 0.00068 & 0.00145 \\
\hline Average & $\begin{array}{l}\text { Stnd Dev } \\
\text { (each axis) }\end{array}$ & 0.00049 & 0.00045 & 0.00032 & 0.00220 & 0.00144 & 0.00289 \\
\hline Average & $\begin{array}{l}\text { Stnd Dev } \\
\text { (all axes) }\end{array}$ & & 0.00042 & & & 0.00218 & \\
\hline
\end{tabular}

Figure 7: Table of Standard Deviations found from measurements by 2 different operators. All values in inches.

For the T-bar data, the 5 measured points per target are averaged for a single point for the laser tracker data. This data is then compared to the mechanical calibration measurements using a best-fit transformation. The transformation has an uncertainty of less than 0.001 ", obtained from the residuals between the transformed laser tracker points and the measured mechanical data. The newly measured laser tracker data thus agrees with the reference data points to within $0.001 "$.

A similar analysis is then performed between the cathetometer data and the transformed laser tracker points. After its 5 measurements per target are analyzed to provide 6 averaged points, the cathetometer data is then best-fit transformed to the laser tracker measurements. The laser tracker data, instead of the mechanical data, is used because two of the points necessary for the transformation are the newly added T-bar targets. As the laser tracker data matches the mechanical data within 0.001 ", it can reliably serve as a reference for the cathetometer measurements.

Figure 8 shows the results from the best-fit transformation for the T-bar data. The standard deviation of the residuals differs widely from axis to axis, but all are within the required limit. The $\mathrm{X}$ axis, with an uncertainty of 0.005 ", is the furthest from nominal, while the Y axis, with an uncertainty of 0.0026 ", is the closest. 


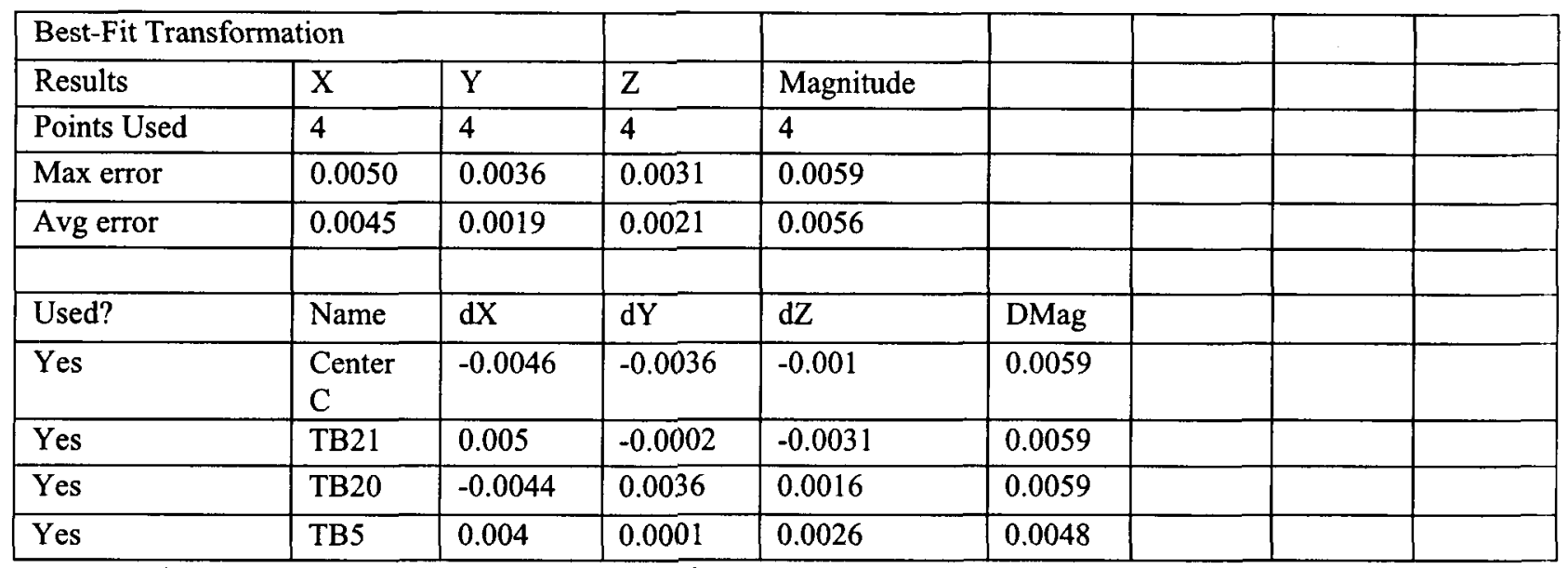

Figure 8: Results for Best-Fit Transform of Cathetometer Data to Laser Tracker Data for T-bar. All values in inches.

As no mechanical data exists for the points added to the Surrogate Wide Field, a single best-fit transformation is performed to compare the cathetometer to the laser tracker data. Figure 9 shows the results from this transform using the 4 best points. The uncertainty in each axis is less than that of the T-bar data: in the $\mathrm{X}$ axis it is 0.0021 ", in the $\mathrm{Y}$ axis it is 0.0023 ", and the $Z$ axis is $0.00055^{\prime \prime}$. This is well within the requirement.

\begin{tabular}{|l|l|l|l|l|l|}
\hline Best-Fit Transformation & & & & \\
\hline Results & $\mathrm{X}$ & $\mathrm{Y}$ & $\mathrm{Z}$ & Magnitude & \\
\hline Points Used & 4 & 4 & 4 & 4 & \\
\hline Max error & 0.0038 & 0.0038 & 0.0009 & 0.0047 & \\
\hline Avg error & 0.0021 & 0.0023 & 0.00055 & 0.0036 & \\
\hline & & & & & \\
\hline Used? & Name & $\mathrm{dX}$ & $\mathrm{dY}$ & $\mathrm{dZ}$ & DMag \\
\hline Yes & Surrogate 5 & 0.0015 & 0.0017 & 0.0002 & 0.0023 \\
\hline Yes & Surrogate 3 & -0.0004 & 0.0031 & -0.0007 & 0.0032 \\
\hline Yes & Surrogate 2 & -0.0038 & -0.0009 & 0.0009 & 0.0040 \\
\hline Yes & Surrogate 6 & 0.0027 & -0.0038 & -0.0004 & 0.0047 \\
\hline
\end{tabular}

Figure 9: Best-Fit Transformation Results of Cathetometer to Laser Tracker Data on Surrogate Camera. All values in inches.

\section{CONCLUSIONS}

The cathetometer metrology system for the Wide Field Camera 3 Project has been fully calibrated and is ready for use. The calibration methodology chosen has been justified by comparison of cathetometer measurements to known references. The uncertainty of the cathetometers derived from these measurements ranges from 0.002 " to 0.005 " for each axis, with an average value of 0.003 ". This is within the requirements set for these cathetometers, and is comparable to other metrology systems (laser trackers, theodolites) currently in use. The low-manpower and low-cost approach taken has saved approximately $\$ 500,000$ or more in providing an essential metrology tool for the completion of WFC3 instrument for the Hubble Space Telescope.

\section{ACKNOWLEDGEMENTS}

The author would like to thank the following people for their assistance in the completion of the above work: Kevin Redman, Vicki Roberts, Dean Osgood, Lou Worrel, and Carlos Aviado of NSI; Henry Sampler and Bill Eichhorn of NASA/GSFC, Larry Sedley of Compumotor, and Beau Legeer of RSI. 


\section{REFERENCES}

1. Numerical Recipes in Fortran, $2^{\text {nd }}$ ed., William H. Press et. al., Cambridge University Press, 1992. 